\title{
THE STRUCTURE OF SUBSTITUTION MINIMAL SETS
}

\author{
BY \\ ETHAN M. COVEN AND MICHAEL S. KEANE( $\left.{ }^{1}\right)$
}

\begin{abstract}
Substitutions of constant length on two symbols and their corresponding minimal dynamical systems are divided into three classes: finite, discrete and continuous. Finite substitutions give rise to uninteresting systems. Discrete substitutions yield strictly ergodic systems with discrete spectra, whose topological structure is determined precisely. Continuous substitutions yield strictly ergodic systems with partly continuous and partly discrete spectra, whose topological structure is studied by means of an associated discrete substitution. Topological and measure-theoretic isomorphisms are studied for discrete and continuous substitutions, and a complete topological invariant, the normal form of a substitution, is given.
\end{abstract}

0 . Introduction. Let $\theta$ denote a substitution of constant length $n$ on the symbols 0 and 1 . Our main objective is the classification of the dynamical systems $\left(\mathcal{O}_{\theta}, \sigma\right)$ arising from such substitutions. A substitution $\theta$ is either finite, discrete, or continuous as defined in $\S 3$.

Finite substitutions give rise to dynamical systems all of whose minimal sets are periodic orbits. Discrete and continuous substitutions both give rise to strictly ergodic dynamical systems with discrete and partially continuous spectra respectively. More specifically, if $\theta$ is discrete, then $\mathcal{O}_{\theta}$ is the orbit-closure of a Toeplitz bisequence, and if $\theta$ is continuous, then $\mathcal{O}_{\theta}$ is the orbit-closure of an extended generalized Morse sequence. Dynamical systems belonging to discrete substitutions are measure-theoretically isomorphic if and only if the lengths of the substitutions have the same prime factors.

If $\theta$ is discrete, we are able to give an explicit construction of $\left(\mathcal{O}_{\theta}, \sigma\right)$ as an almost one-to-one extension of the $n$-adic system $(Z(n), \tau)$. We associate with $\theta$ an object called a group system which is a complete invariant for topological isomorphism of $\left(\mathcal{O}_{\theta}, \sigma\right)$. The concept of normal form for a discrete substitution is defined and it is shown that discrete substitutions of the same length possess topologically isomorphic dynamical systems if and only if they have the same normal form.

To each continuous substitution $\theta$ we associate a discrete substitution $\bar{\theta}$ of the same length such that $\left(\mathcal{O}_{\theta}, \sigma\right)$ is a distal extension of $\left(\mathcal{O}_{\bar{\theta}}, \sigma\right)$. Each fibre consists of two points which are mirror images of each other. A normal form for continuous

Received by the editors May 22, 1970.

AMS 1970 subject classifications. Primary 28A65, 54H20.

Key words and phrases. Shift dynamical system, $n$-adic system, strict ergodicity, substitution, substitution minimal set, structure system, group system, normal form.

(1) The authors were partially supported by NSF Grants GP-23105 and GP-9039.

Copyright (C) 1972, American Mathematical Society 
substitutions is defined which yields an isomorphism theorem analogous to the discrete case.

We remark that our definition of substitution is quite restrictive. The construction given in this paper will generalize to some substitutions of constant length on more than two symbols, although none of the ideas appears to be useful in dealing with substitutions of nonconstant length.

1. Basic concepts. Let $X$ be a nonempty compact Hausdorff space and let $\varphi$ be a homeomorphism of $X$ onto itself. The pair $(X, \varphi)$ is called a dynamical system. We say that $(Y, \varphi)$ is a subsystem of $(X, \varphi)$ if $Y$ is a nonempty compact subset of $X$ which is invariant under $\varphi$. A dynamical system is called minimal if it contains no proper subsystem. For each point $x \in X$, the orbit of $x$ is the set defined by $\mathcal{O}(x)$ $=\left\{\varphi^{k}(x) \mid k=0, \pm 1, \pm 2, \ldots\right\}$. The system $(X, \varphi)$ is minimal if and only if the orbit of each point is dense in $X$. Other criteria for minimality may be found in [3].

We shall be interested in minimal subsystems of the dynamical system defined below. Let

$$
\Omega=\prod_{-\infty}^{\infty}\{0,1\}=\left\{\omega=\left(\cdots \omega_{-1} \omega_{0} \omega_{1} \cdots\right) \mid \omega_{i}=0 \text { or } 1\right\} .
$$

Given the product topology, $\Omega$ is a compact metrizable space homeomorphic to the Cantor discontinuum. A compatible metric is given by $d\left(\omega, \omega^{\prime}\right)=1 /(n+1)$ where $n$ is the largest nonnegative integer such that $\omega_{i}=\omega_{i}^{\prime}$ for $|i|<n$. Let the shift $\sigma: \Omega \rightarrow \Omega$ be defined by

$$
\sigma(\omega)_{i}=\omega_{i+1} \quad(i=0, \pm 1, \pm 2, \ldots) .
$$

We call $(\Omega, \sigma)$ the shift dynamical system. The points of $\Omega$ are called bisequences. We shall also talk about sequences (one-sided strings of zeros and ones) and blocks (finite strings of zeros and ones).

If $\omega \in \Omega, k$ and $m$ integers with $m$ positive, then $\omega[k, m]$ will denote the $m$-block occurring in $\omega$ starting at place $k$. That is, if $\omega=\left(\cdots \omega_{-1} \omega_{0} \omega_{1} \cdots\right)$, then $\omega[k, m]$ $=\omega_{k} \omega_{k+1} \cdots \omega_{k+m-1}$. Similarly we denote by $b[k, m]$ the $m$-block starting at place $k$ in $b$ where $b$ is a sequence or block, whenever this definition makes sense. If $b$ is a block, sequence or bisequence, then the mirror $\tilde{b}$ of $b$ is the block, sequence or bisequence obtained from $b$ by replacing all the zeros in $b$ by ones and all the ones by zeros. For example, $(011)^{\sim}=100$.

Let $(X, \varphi)$ and $(Y, \psi)$ be dynamical systems. A homomorphism of $(X, \varphi)$ onto $(Y, \dot{\psi})$ is a continuous map $\pi: X \rightarrow Y$ which is equivariant in the sense that $\pi \varphi=\psi \pi$. A homomorphism which is one-to-one is called an isomorphism.

A dynamical system $(X, \varphi)$ is called equicontinuous if the set of maps $\left\{\varphi^{n} \mid n=0, \pm 1, \pm 2, \ldots\right\}$ is uniformly equicontinuous. Every dynamical system has a "largest" equicontinuous homomorphic image called its structure system. Here "largest" means that any equicontinuous homomorphic image of the original system is a homomorphic image of the structure system. The structure system is sometimes called the equicontinuous structure transformation group [1]. 
The system $(X, \varphi)$ is called uniquely ergodic if there is exactly one $\varphi$-invariant probability measure on the Borel sets of $X$. If $(X, \varphi)$ is uniquely ergodic, then its unique invariant measure is ergodic with respect to $\varphi$, that is $X$ cannot be written as the disjoint union of two $\varphi$-invariant subsets each having positive measure. A uniquely ergodic system which is minimal is called strictly ergodic. Criteria for unique and strict ergodicity may be found in [9].

Let $(X, \varphi)$ and $(Y, \psi)$ be uniquely ergodic with invariant measures $\mu$ and $\nu$ respectively. An equivariant map $\pi:(X, \varphi) \rightarrow(Y, \psi)$ is called a measure-theoretic isomorphism if there is an invariant subset $X^{\prime}$ of $X$ such that $\mu\left(X^{\prime}\right)=1, \pi$ is one-toone on $X^{\prime}$ and $\nu(\pi(E))=\mu(E)$ for every Borel set $E$ contained in $X^{\prime}$. A homomorphism $\pi:(X, \varphi) \rightarrow(Y, \psi)$ is a measure-theoretic isomorphism if there is an invariant subset $Y^{\prime}$ of $Y$ such that $\nu\left(Y^{\prime}\right)=1$ and $\pi$ is one-to-one on $\pi^{-1}\left(Y^{\prime}\right)$. In particular every isomorphism is a measure-theoretic isomorphism.

The homeomorphism $\varphi$ induces a unitary operator on $L^{2}(X, \mu)$ by $f \rightarrow f \circ \varphi$. It is customary to refer to the spectrum of this operator as the spectrum of $(X, \varphi)$. The spectral properties of $(X, \varphi)$ are invariant under isomorphisms of $(X, \varphi)$.

The strictly ergodic systems with which we shall be dealing are certain subsystems of $(\Omega, \sigma)$, as well as the systems described below.

For $n \geqq 2$, let $Z(n)$ denote the additive group of $n$-adic integers

$$
Z(n)=\left\{\sum_{i=0}^{\infty} z_{i} n^{i} \mid z_{i}=0,1, \ldots, n-1\right\} .
$$

With a neighborhood base at 0 consisting of sets of the form

$$
U_{k}=\left\{\sum u_{i} n^{i} \mid u_{i}=0 \text { for } i<k\right\} \quad(k=0,1,2, \ldots),
$$

$Z(n)$ is a compact abelian group. With $\tau: Z(n) \rightarrow Z(n)$ defined by $\tau(z)=z+1$, we call $(Z(n), \tau)$ the $n$-adic system. This system is strictly ergodic and has discrete spectrum, the invariant measure being normalized Haar measure. Two such systems $(Z(n), \tau)$ and $(Z(m), \tau)$ are isomorphic if and only if $m$ and $n$ have the same prime factors.

Every element $z$ of the orbit of 0 in $Z(n)$ is of the form $z=\sum z_{i} n^{i}$ where either $z_{i}=0$ for all but finitely many $i$, or $z_{i}=n-1$ for all but finitely many $i$. We shall regard such elements both as ordinary integers and as elements of $Z(n)$.

2. Some special bisequences. Let $a=a_{0} a_{1} \cdots a_{m-1}$ be an $m$-block and $b=b_{0} b_{1}$ $\cdots b_{n-1}$ be an $n$-block. We define $a \times b=a^{b_{0}} a^{b_{1}} \cdots a^{b_{n-1}}$ where $a^{0}=a$ and $a^{1}=\tilde{a}$. Then $a \times b$ is an $m n$-block and the operation is associative. See [6] for further details.

Suppose that $b=b_{0} b_{1} \cdots b_{n-1}$ is an $n$-block with $b_{0}=0$ and $n \geqq 2$. Then for any block $a$, the block $a \times b$ begins with the block $a$. Therefore we may define a sequence $s=\left(s_{0} s_{1} \cdots\right)$ by

$$
s\left[0, n^{k}\right]=b \times b \times \cdots \times b \quad(k \text { times })
$$


for $k=1,2, \ldots$. A sequence obtained in this way is an example of a generalized Morse sequence. The well-known classical Morse sequence is generated by $b=01$. If $b \neq 00 \cdots 0$ or $0101 \cdots 010$, then $s$ may be extended to the left in two ways to form bisequences $\omega$ and $\omega^{\prime}$ with minimal orbit-closures. This is done by defining $\omega\left[-n^{2 k}, n^{2 k}\right]=s\left[0, n^{2 k}\right]$ and $\omega^{\prime}\left[-n^{2 k}, n^{2 k}\right]=\tilde{s}\left[0, n^{2 k}\right]$ for $k=1,2, \ldots$

THEOREM [6]. The orbit-closures of $\omega$ and $\omega^{\prime}$ are equal. The system $(\mathrm{cl} O(\omega), \sigma)$ is strictly ergodic with partly discrete and partly continuous spectrum.

A point $\omega \in \Omega$ is called a Toeplitz bisequence if there is a collection of pairwise disjoint arithmetic progressions $\left\{T_{i}\right\}$ whose union is the set of all integers such that $\omega_{n}=\omega_{m}$ if $n$ and $m$ belong to the same $T_{i}$. A Toeplitz bisequence is called regular if $\sum 1 / p_{i}=1$ where $T_{i}=\left\{m_{i}+k p_{i} \mid k=0, \pm 1, \pm 2, \ldots\right\}$ and $p_{i}>0$.

THEOREM [5]. If $\omega$ is a Toeplitz bisequence, then $(\mathrm{cl} \mathcal{O}(\omega), \sigma)$ is minimal. If in addition, $\omega$ is regular, then $(\mathrm{cl} \mathcal{O}(\omega), \sigma)$ is strictly ergodic and has purely discrete spectrum.

We remark that not all Toeplitz bisequences are regular. The bisequence described by Oxtoby $[9, \mathrm{pp} .135-136]$ is an example.

3. Substitutions. Let $n$ be an integer, $n \geqq 2$. A substitution of length $n$ is a map $\theta$ from $\{0,1\}$ to the set of $n$-blocks, that is $\theta$ is a pair of $n$-blocks $\theta(0)=a_{0} a_{1} \cdots a_{n-1}$, $\theta(1)=b_{0} b_{1} \cdots b_{n-1}$. Our definition is less general than the usual one, see [2].

A substitution generates, in a natural way, maps from blocks to blocks, sequences to sequences and bisequences to bisequences. We shall use the symbol $\theta$ to denote these maps as well. For example, if $\omega=\left(\cdots \omega_{-1} \omega_{0} \omega_{1} \cdots\right) \in \Omega$, then $\theta(\omega)=\omega^{\prime}$ is given by $\omega^{\prime}[k n, n]=\theta\left(\omega_{k}\right)(k=0, \pm 1, \pm 2, \ldots)$. In particular $\theta: \Omega \rightarrow \Omega$ is continuous. A substitution $\theta$ generates a substitution $\theta^{2}$ of length $n^{2}$ by $\theta^{2}(i)$ $=\theta(\theta(i))$ for $i=0,1$. Similarly, for each positive integer $k, \theta^{k}$ is a substitution of length $n^{k}$.

We shall divide substitutions into three classes, whose names are derived from the spectral properties of the dynamical systems associated with them.

$\theta$ is called finite if any of the following conditions hold:

(1) $a_{i}=b_{i}$ for all $i$.

(2) $a_{i}=0$ for all $i$ or $b_{i}=1$ for all $i$.

(3) $a_{i}=1$ for all $i$ and $b_{i}=0$ for all $i$.

(4) $n$ is odd and $a=\tilde{b}=0101 \cdots 010$ or $1010 \cdots 101$.

$\dot{\theta}$ is called continuous if it is not finite and if $a=\tilde{b}$.

$\theta$ is called discrete if it is neither finite nor continuous.

A substitution is discrete if and only if both $I_{1}=\left\{i \mid a_{i}=b_{i}\right\}$ and $J_{1}=\left\{i \mid a_{i} \neq b_{i}\right\}$ are nonempty, that is $\theta(0)$ and $\theta(1)$ agree at some but not every place. Note that $\theta$ and $\theta^{k}$ are in the same class for all $k \geqq 1$.

As in [2] a substitution $\theta$ of length $n$ defines a map $\lambda$ on the set of 2-blocks by

$$
00 \rightarrow a_{n-1} a_{0}, \quad 01 \rightarrow a_{n-1} b_{0}, \quad 10 \rightarrow b_{n-1} a_{0}, \quad 11 \rightarrow b_{n-1} b_{0} .
$$


It is easily seen that the map $\lambda^{2}$ (which corresponds to the substitution $\theta^{2}$ ) has either one, two or four fixed points, depending on whether $a$ and $b$ agree at both ends, one end or neither end. If $p q$ is such a fixed point, then $\theta^{2}$ is a contraction on the closed set $\left\{\omega \in \Omega \mid \omega_{-1} \omega_{0}=p q\right\}$. Therefore there is a unique point $\omega \in \Omega$ which satisfies $\theta^{2}(\omega)=\omega$ and $\omega_{-1} \omega_{0}=p q$. We shall denote this point by $\omega^{p q}$.

THEOREM [2], [7]. Let $\theta$ be a continuous or discrete substitution. There is a minimal subsystem $\left(\mathcal{O}_{\theta}, \sigma\right)$ of $(\Omega, \sigma)$ such that for any pq invariant under $\lambda^{2}, \mathcal{O}_{\theta}=\operatorname{cl} \mathcal{O}\left(\omega^{p q}\right)$. Furthermore, $\left(\mathcal{O}_{\theta}, \sigma\right)$ is a strictly ergodic system.

The first statement is proved in [2], the second in [7]. We shall call $\mathcal{O}_{\theta}$ a substitution minimal set. Note that $\mathcal{O}_{\theta}=\mathcal{O}_{\theta^{k}}$ for all $k \geqq 1$.

If $\theta$ is finite, then the only minimal sets contained in $\mathrm{cl} \mathcal{O}\left(\omega^{p q}\right)$ are periodic orbits. Since we consider periodic orbits to be uninteresting as minimal sets, we shall confine our attention to continuous and discrete substitutions.

4. Measure-theoretic classification. In this section we show that a substitution minimal set obtained from a continuous substitution cannot be isomorphic to one obtained from a discrete substitution.

THEOREM. If $\theta$ is continuous, then $\mathcal{O}_{\theta}$ is the orbit-closure of an extended generalized Morse sequence. If $\theta$ is discrete, then $\mathcal{O}_{\theta}$ is the orbit-closure of a regular Toeplitz bisequence.

Proof. Let $\theta$ be continuous. If $a_{0}=0$, let $s=a \times a \times \cdots$, and if $a_{0}=1$, let $s=b \times b \times \cdots$. It is easily verified that if $s$ is extended to the left as in $\S 2$, then a bisequence is obtained whose orbit-closure is $\mathcal{O}_{\theta}$.

Let $\theta$ be discrete and have length $n$. By replacing $\theta$ by $\theta^{2}$ if necessary, we may assume that $a_{i}=b_{i}$ for some $i \neq 0, n-1$. The map $\sigma^{i} \theta$ is a contraction on the closed set $\left\{\omega \in \Omega \mid \omega_{0}=a_{i}\right\}$ and there is a unique point $\omega \in \Omega$ such that $\omega_{0}=a_{i}$ and $\sigma^{i} \theta(\omega)$ $=\omega$. Now

$$
\begin{aligned}
\omega[-i, n] & =\theta\left(a_{i}\right), \\
\omega\left[-i-i n, n^{2}\right] & =\theta^{2}\left(a_{i}\right), \\
& \vdots \\
\omega\left[-\sum_{j=0}^{k-1} i n^{j}, n^{k}\right] & =\theta^{k}\left(a_{i}\right) \quad(k=1,2, \ldots) .
\end{aligned}
$$

Since $i \neq 0$ or $n-1$, the integers $\sum_{j=0}^{k-1} i n^{j}$ and $n^{k}-\sum_{j=0}^{k-1} i n^{j}$ both increase with $k$, therefore $\omega \in \mathcal{O}_{\theta}$ and $\mathcal{O}_{\theta}=\operatorname{cl} \mathcal{O}(\omega)$.

We claim that $\omega$ is a regular Toeplitz bisequence. Since $a_{i}=b_{i}$ and $\omega$ is invariant under $\sigma^{i} \theta, \omega_{k n}=a_{i}$ for $k=0, \pm 1, \pm 2, \ldots$ We let $T_{1}=\{k n \mid k=0, \pm 1, \pm 2, \ldots\}$, an arithmetic progression with period $n$. Applying $\sigma^{i} \theta$ again, we see that the $n$-block $\theta\left(a_{i}\right)$ appears in $\omega$ with period $n^{2}$, starting at places $-i+k n^{2}(k=0, \pm 1, \pm 2, \ldots)$. We set $T_{2}, T_{3}, \ldots, T_{n}$ equal to $\left\{-i+k n^{2}\right\},\left\{-i+1+k n^{2}\right\}, \ldots,\left\{-i+n-1+k n^{2}\right\}$ omitting $\left\{k n^{2}\right\}$. Thus we have defined $n-1$ arithmetic progressions of period $n^{2}$. 
Continuing in this manner, we get $(n-1)^{2}$ arithmetic progressions of period $n^{3}$, $(n-1)^{3}$ arithmetic progressions of period $n^{4}$, etc. Every integer appears in exactly one of these progressions and $1 / n+(n-1) / n^{2}+(n-1)^{2} / n^{3}+\cdots=1$. Therefore $\omega$ is a regular Toeplitz bisequence.

COROllary. Every substitution minimal set is strictly ergodic. If $\theta$ is continuous and $\zeta$ is discrete, then the systems $\left(\mathcal{O}_{\theta}, \sigma\right)$ and $\left(\mathcal{O}_{\zeta}, \sigma\right)$ are not isomorphic.

Proof. By the results of $\S 2,\left(\mathcal{O}_{\theta}, \sigma\right)$ has partly continuous spectrum while $\left(\mathcal{O}_{\zeta}, \sigma\right)$ has purely discrete spectrum. Therefore they are not measure-theoretically isomorphic and hence not isomorphic.

5. The structure system of a discrete substitution. Let $\theta$ be a discrete substitution of length $n$ with $\theta(0)=a, \theta(1)=b$. It is known [2] that the structure system of $\left(\mathcal{O}_{\theta}, \sigma\right)$ is the $n$-adic system $(Z(n), \tau)$. In this section we shall give an explicit construction of the homomorphism $\pi:\left(\mathcal{O}_{\theta}, \sigma\right) \rightarrow(Z(n), \tau)$, and show that $\pi$ is a measuretheoretic isomorphism.

LEMMA 1. There exists an integer $k \geqq 1$ such that $\theta^{k}(0)$ and $\theta^{k}(1)$ both contain 00,01 and 10, or both contain 11, 01 and 10 .

Proof. Either $\theta(0)$ or $\theta(1)$ contains both 0 and 1. By examining cases, it is easy to see that $\theta^{2}(0)$ and $\theta^{2}(1)$ both contain 01 and 10 . Either $\theta^{2}(0)$ or $\theta^{2}(1)$ contains 00 or 11 , for otherwise $n$ is odd and $a=\tilde{b}=0101 \cdots 010$ or $1010 \cdots 101$. The proof is completed by the observation that both $\theta^{3}(0)$ and $\theta^{3}(1)$ contain every 2-block which appears in $\theta^{2}(0)$ or in $\theta^{2}(1)$.

Note that if $\theta(0)=001$ and $\theta(1)=010$, then no power of $\theta$ contains 11 .

LEMMA 2. If each of the blocks $a a[k, n], a b[k, n]$ and $b a[k, n]$ is either $a$ or $b$, then $k=0$ or $n$.

Proof. Suppose $0<k<n$. Then $a b[k, n] \neq b a[k, n]$, for otherwise we would have $a=b$. The bisequence $(\cdots a b a b \cdots)$ is invariant under shifts of $2 n$ and either $k$ or $k+n$. Therefore it has period either $\operatorname{GCD}(k, 2 n)$ or $\operatorname{GCD}(k+n, 2 n)$. Both of these numbers may be expressed as $2^{\alpha} p$ where $\alpha=0$ or 1 and $p$ divides $n$. If $\alpha=0$, then we again get $a=b$. If $\alpha=1$, then there exist $p$-blocks $c$ and $d$ such that $a=c d c d$ $\cdots c d c$ and $b=d c d c \cdots d c d$. Since $a a[k, n]$ is either $a$ or $b, c=d$ which again yields $a=b$.

The lemma also holds with $b b$ in place of $a a$.

Lemma 3. Let pq be a 2-block which is invariant under $\lambda^{2}$. Suppose that $\omega \in \Omega$ and that $\left\{k_{i}\right\}$ is a sequence of integers such that

$$
\lim _{i \rightarrow \infty} \sigma^{k_{i}}\left(\omega^{p q}\right)=\omega .
$$

Then for each positive integer $j$, there is an integer $N_{j}$ such that $i, i^{\prime} \geqq N_{j}$ implies $k_{i} \equiv k_{i}, \bmod n^{i}$. 
Proof. Since $\mathcal{O}_{\theta}=\mathcal{O}_{\theta}$, we may assume that $j=1$, hence by Lemma 1 , that $\theta(0)$ and $\theta(1)$ both contain 01,10 and either 00 or 11 . Suppose that 00 appears in $\theta(0)$ and $\theta(1)$. Since $\omega \in \mathcal{O}_{\theta}$, it contains the same finite blocks as $\omega^{p q}$. Therefore for some $m, \omega[0, m]$ contains the blocks $a a, a b$ and $b a$ starting at places which differ by multiples of $n$. The bisequence $\omega^{p q}$ may be grouped so that each $n$-block is either $a$ or $b$, and for large enough $i, \sigma^{k_{i}} \omega^{p q}[0, m]=\omega[0, m]$. Therefore by Lemma 2, for large $i$ the value of $k_{i} \bmod n$ is unique. A similar proof applies if 11 appears in $\theta(0)$ and $\theta(1)$.

We define the map $\pi: \mathcal{O}_{\theta} \rightarrow Z(n)$ by

$$
\pi(\omega)=\lim _{i \rightarrow \infty} \tau^{k_{i}}(0)
$$

where $\left\{k_{i}\right\}$ is chosen so that $\sigma^{k_{i}} \omega^{p q} \rightarrow \omega$ for some $p q$ invariant under $\lambda^{2}$. Lemma 3 shows that the map is well defined. If $d\left(\omega, \omega^{\prime}\right)<1 /(m+1)$, then $\omega[0, m]=\omega^{\prime}[0, m]$. Therefore the proof of Lemma 3 also shows that $\pi$ is continuous. It is clear that $\pi \sigma=\tau \pi$. Since $\pi$ maps $\omega^{p q}$ to 0 , it takes $\mathcal{O}\left(\omega^{p q}\right)$ to the orbit of 0 in $Z(n)$. Thus we have proved the following:

THEOREM 1. There is a homomorphism $\pi:\left(\mathcal{O}_{\theta}, \sigma\right) \rightarrow(Z(n), \tau)$ taking each $\theta^{2}$ invariant point to 0 . Furthermore, if $\pi(\omega)=\sum z_{i} n^{i}$, then for each $k \geqq 1$, $\omega\left[-\sum_{i=0}^{k-1} z_{i} n^{i}, n^{k}\right]$ is either $\theta^{k}(0)$ or $\theta^{k}(1)$.

Our next goal is to determine the set on which $\pi$ is one-to-one. First we introduce the following notation.

If $z=\sum z_{i} n^{i}$, let $z^{(k)}$ denote the $k$ th partial sum of $z$, that is $z^{(k)}=\sum_{i=0}^{k-1} z_{i} n^{i}$. Then $z^{(k)}$ is an ordinary integer and $z^{(k)} \rightarrow z$.

For each $k \geqq 1$, let

$$
\begin{aligned}
& I_{k}=\left\{m \mid 0 \leqq m \leqq n^{k}-1, \theta^{k}(0) \text { and } \theta^{k}(1) \text { agree at place } m\right\}, \\
& J_{k}=\left\{m \mid 0 \leqq m \leqq n^{k}-1, \theta^{k}(0) \text { and } \theta^{k}(1) \text { disagree at place } m\right\} .
\end{aligned}
$$

An easy induction argument shows that

$$
I_{k}=\left\{\sum_{i=0}^{k-1} z_{i} n^{i} \mid z_{i} \in I_{1} \text { for some } i\right\}, \quad J_{k}=\left\{\sum_{i=0}^{k-1} z_{i} n^{i} \mid z_{i} \in J_{1} \text { for all } i\right\} .
$$

Let

$$
\begin{aligned}
I_{\infty} & =\left\{\sum_{i=0}^{\infty} z_{i} n^{i} \mid z_{i} \in I_{1} \text { for some } i\right\}, & J_{\infty} & =\left\{\sum_{i=0}^{\infty} z_{i} n^{i} \mid z_{i} \in J_{1} \text { for all } i\right\}, \\
E & =\bigcup\left\{\tau^{k} J_{\infty} \mid k=0, \pm 1, \pm 2, \ldots\right\}, & Z^{*} & =Z(n)-E .
\end{aligned}
$$

Then $J_{\infty}$ is closed and nowhere dense. Hence $Z^{*}$ is a $G_{\delta}$ set which, by the Baire Category Theorem, is dense in $Z(n)$.

THEOREM 2. Let $\nu$ denote normalized Haar measure on $Z(n)$. Then $\nu\left(Z^{*}\right)=1$.

Proof. It suffices to show that $\nu\left(I_{\infty}\right)=1$, for then both $J_{\infty}$ and $E$ have measure zero. Note that $I_{\infty}=\bigcup_{k=0}^{\infty} B_{k}$ where $B_{k}=\left\{\sum z_{i} n^{i} \mid z_{i} \in J_{1}\right.$ for $i<k$ and $\left.z_{k} \in I_{1}\right\}$ and 
that the collection $\left\{B_{k}\right\}$ is pairwise disjoint. If $J_{1}$ has $m$ elements, then $\nu\left(B_{k}\right)$ $=(m / n)^{k}(n-m) / n$. Therefore $\nu\left(I_{\infty}\right)=((n-m) / n) \sum_{k=0}^{\infty}(m / n)^{k}=1$.

THEOREM 3. $Z^{*}=\left\{z \in Z(n) \mid \pi^{-1}(z)\right.$ is a single point $\}$. If $z \in E$, then $\pi^{-1}(z)$ consists of exactly two points unless $z \in \mathcal{O}(0)$ and $a$ and $b$ disagree at both endpoints. In this case, $\pi^{-1}(z)$ consists of four points.

Proof. Let $z \in Z(n)$. If $\omega \in \pi^{-1}(z)$, then by Theorem $1, \omega\left[-z^{(k)}, n^{k}\right]$ is either $\theta^{k}(0)$ or $\theta^{k}(1)$. In particular, $\omega_{0}$ is the symbol at the $z^{(k)}$ th place of either $\theta^{k}(0)$ or $\theta^{k}(1)$.

If $z \in Z^{*}$, then $z^{(k)} \in I_{k}$ for all large $k$ and $\omega_{0}$ is uniquely determined. Since $Z^{*}$ is $\tau$-invariant and $\sigma^{m} \omega \in \pi^{-1}\left(\tau^{m} z\right), \omega_{m}=\left(\sigma^{m} \omega\right)_{0}$ is uniquely determined for all $m$. Thus $\pi^{-1}(z)$ is a singleton.

Suppose next that $z \in E$ but $z \notin \mathcal{O}(0)$. Since both $E$ and $\mathcal{O}(0)$ are $\tau$-invariant, we may assume that $z \in J_{\infty}$. Both $z^{(k)}$ and $n^{k}-z^{(k)}$, regarded as ordinary integers, increase with $k$. Thus there are points $\omega, \omega^{\prime} \in \mathcal{O}_{\theta}$ such that for each $k \geqq 1$,

$$
\left\{\omega\left[-z^{(k)}, n^{k}\right], \omega^{\prime}\left[-z^{(k)}, n^{k}\right]\right\}=\left\{\theta^{k}(0), \theta^{k}(1)\right\} .
$$

Since $z \in J_{\infty}, \omega_{0} \neq \omega_{0}^{\prime}$ and hence $\omega \neq \omega^{\prime}$. Theorem 1 shows that $\pi^{-1}(z)=\left\{\omega, \omega^{\prime}\right\}$.

Suppose that $z \in E \cap \mathcal{O}(0)$. We may assume that $z=0$. Recall that $\pi^{-1}(0)$ $=\left\{\omega^{p q} \mid p q\right.$ invariant under $\left.\lambda^{2}\right\}$. Therefore $\pi^{-1}(z)$ consists of two or four points depending on whether $a$ and $b$ disagree at one end or at both ends.

CoRollaRy. The map $\pi:\left(\mathcal{O}_{\theta}, \sigma\right) \rightarrow(Z(n), \tau)$ is a measure-theoretic isomorphism. Hence two discrete substitutions have measure-theoretically isomorphic minimal sets if and only if their lengths have the same prime factors.

This result follows immediately from the last three theorems. The fact that $\left(\mathcal{O}_{\theta}, \sigma\right)$ and $(Z(n), \tau)$ are measure-theoretically isomorphic was first observed by Neveu [8].

The situation with continuous substitutions is quite different. By analysis of the continuous part of the spectrum, it can be shown that two continuous substitutions have measure-theoretically isomorphic minimal sets only if the substitutions have a common power.

6. The group system of a discrete substitution. As in $\S 5$, let $\theta$ be a discrete substitution of length $n$ and let $\pi:\left(\mathcal{O}_{\theta}, \sigma\right) \rightarrow(Z(n), \tau)$ be the homomorphism described there. Define $p_{0}: Z^{*} \rightarrow\{0,1\}$ by $p_{0}(z)=\omega_{0}$ if $\pi(\omega)=z$. Then $p_{0}$ is defined and continuous on $Z^{*}$. The triple $\left((Z(n), \tau), Z^{*}, p_{0}\right)$ will be called the group system of $\theta$. Our main goal in this section will be to construct the system $\left(\mathcal{O}_{\theta}, \sigma\right)$ from the group system of $\theta$.

Let $\mathscr{C}$ be the algebra of all complex-valued functions on $Z^{*}$ which are restrictions to $Z^{*}$ of functions continuous on $Z(n)$, that is $\mathscr{C}=\left\{f \mid Z^{*}: f \in \mathscr{C}(Z(n))\right\}$. For each integer $k$, define $p_{k}: Z^{*} \rightarrow\{0,1\}$ by $p_{k}(z)=p_{0}\left(\tau^{k} z\right)$. Then every $p_{k}$ is continuous on $Z^{*}$. Let $\mathscr{B}$ be the Banach algebra (sup-norm) generated by $\mathscr{C}$ and

$$
\left\{p_{k} \mid k=0, \pm 1, \pm 2, \ldots\right\} \text {. }
$$


Note that $\mathscr{B}$ is a closed subalgebra of the bounded continuous functions on $Z^{*}$. Let $X$ be the maximal ideal space of $\mathscr{B}$; the elements of $X$ may be identified with the nonzero multiplicative functionals on $\mathscr{B}$. Since $Z^{*}$ is $\tau$-invariant, $\tau$ induces a homeomorphism of $\mathscr{B}$ by $\tau f(z)=f(\tau z)\left(f \in \mathscr{B}, z \in Z^{*}\right)$, and a homeomorphism $\tau^{*}$ of $X$ by $\tau^{*} x(f)=x(\tau f)(f \in \mathscr{B})$. We claim that $\left(X, \tau^{*}\right)$ is isomorphic to $\left(\mathcal{O}_{\theta}, \sigma\right)$.

Define $\xi$ on $X$ by $\xi(x)_{k}=x\left(p_{k}\right)$. Then $\xi$ maps $X$ into $\Omega$ since $x\left(p_{k}\right)$ must lie in the spectrum of $p_{k}$ which contains only 0 and 1 . It is easy to see that $\xi \tau^{*}=\sigma \xi$ and that $\xi$ is continuous. We must show that $\xi$ is one-to-one and maps $X$ onto $\mathcal{O}_{\theta}$. To do this we will define a homomorphism $\pi^{*}:\left(X, \tau^{*}\right) \rightarrow(Z(n), \tau)$ and show that a sufficient condition for $\xi$ to be one-to-one and map $X$ onto $\mathcal{O}_{\theta}$ is that $\pi^{*-1}\left(Z^{*}\right)$ is dense in $X$. We will then verify that this condition holds.

Define $\pi^{*}: X \rightarrow Z(n)$ by $\pi^{*}(x)=z$ if and only if $x(f)=f(z)$ for all $f \in \mathscr{C}$. This definition makes sense because the elements of $X$ may be viewed as nonzero multiplicative functionals on $\mathscr{C}(Z(n))$ and as such are point-evaluations. It is easy to see that $\pi^{*}$ is a homomorphism of $\left(X, \tau^{*}\right)$ into $(Z(n), \tau)$. Let $X^{*}=\pi^{*-1}\left(Z^{*}\right)$. Then $\xi$ maps $X^{*}$ into $\mathcal{O}_{\theta}$ and the following diagram commutes:

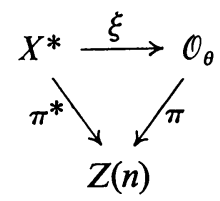

Suppose now that $X^{*}$ is dense in $X$. Then $\xi(X) \subseteq \overline{\mathcal{O}}_{\theta}=\mathcal{O}_{\theta}$. Since $\mathcal{O}_{\theta}$ is minimal, $\xi$ maps $X$ onto $\mathcal{O}_{\theta}$. Furthermore, the following diagram commutes:

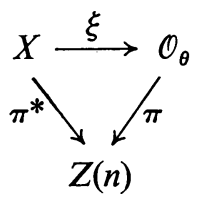

If $x, x^{\prime} \in X$ and $\xi(x)=\xi\left(x^{\prime}\right)$, then $x\left(p_{k}\right)=x^{\prime}\left(p_{k}\right)$ for all $k$. Since the diagram commutes, $\pi^{*}(x)=\pi^{*}\left(x^{\prime}\right)$, that is $x(f)=x^{\prime}(f)$ for all $f \in \mathscr{C}$. Therefore $x(f)=x^{\prime}(f)$ for all $f \in \mathscr{B}$, i.e., $x=x^{\prime}$. Thus $\xi$ is one-to-one.

The remainder of this section will be devoted to showing that $X^{*}$ is dense in $X$. The idea will be to show that $x$ is determined by $\pi^{*}(x)$ when $x \in X^{*}$ and by $\pi^{*}(x)$, $x\left(p_{0}\right)$ and $x\left(p_{-1}\right)$ when $x \notin X^{*}$. For this purpose it will be useful to think of the elements of $\mathscr{C}$ as defined and continuous on $Z(n)$ and to think of $p_{k}$ as defined and continuous on the open set $Z(n)-\tau^{-k} J_{\infty}$.

Lemma. Let $\pi^{*}(x)=z$ and let $f \in \mathscr{B}$ be continuous in some neighborhood of $z$. Then $x(f)=f(z)$.

Proof. Let $N$ be any such neighborhood. Pick $g \in \mathscr{C}(Z(n))$ such that $f$ and $g$ agree on $N$, and $h \in \mathscr{C}(Z(n))$ such that $h(z) \neq 0$ and $h\left(z^{\prime}\right)=0$ if $z^{\prime} \notin N$. Then $h(f-g)$ is identically zero. Therefore $0=x(h(f-g))=h(z)(x(f)-f(z))$ and $x(f)=f(z)$. 
Suppose that $\pi^{*}(x)=z \in Z^{*}$. If $f \in \mathscr{C}$ or if $f=p_{k}$ for some $k$, then $f$ is continuous on an open set containing $z$. By the lemma just proved, $x(f)=f(z)$. Therefore $x(f)=f(z)$ for all $f \in \mathscr{B}$.

Suppose next that $z \notin Z^{*} \cup \mathcal{O}(0)$. We claim that $z$ has exactly two $\pi^{*}$-inverses, corresponding to the two possible values of $p_{k}$, where $z \in \tau^{-k} J_{\infty}$. Since $\tau^{* k} \pi^{*-1}(z)$ $=\pi^{*-1}\left(\tau^{k} z\right)$, we may assume that $z \in J_{\infty}$ and hence that $p_{0}$ is not continuous at $z$. Recall that if $\pi^{*}(x)=z$, then $x$ is determined by $z$ at all $f \in \mathscr{C}$ and at all $p_{k}$ which are continuous at $z$. The following lemma shows that if $p_{k}$ is not continuous at $z$, then $x\left(p_{k}\right)$ is determined by $x\left(p_{0}\right)$.

Lemma. Suppose that $z \in J_{\infty}-\mathcal{O}(0)$ and that neither $p_{0}$ nor $p_{k}$ is continuous at $z$. Then there exists a neighborhood $U$ of 0 in $Z(n)$ such that either $p_{0}(z+u)=p_{k}(z+u)$ for all $u \in U$ such that $z+u \in Z^{*}$ or $p_{0}(z+u)=1-p_{k}(z+u)$ for all $u \in U$ such that $z+u \in Z^{*}$.

In the course of the proof we will make repeated use of the following observation, which is easily verified by induction:

Let $0 \leqq m \leqq n^{i}-1$ and write $m=\sum_{j=0}^{i-1} m_{j} n^{j}$. Then $\theta^{i}(0)$ evaluated at place $m$ is $\theta(s)$ evaluated at $m_{0}$ where $s$ is $\theta^{i-1}(0)$ evaluated at $(1 / n)\left(m-m_{0}\right)$. Furthermore $(1 / n)\left(m-m_{0}\right)=\sum_{j=0}^{i-2} m_{j+1} n^{j}$. A similar statement holds for $\theta^{i}(1)$.

Proof of Lemma. Let $y=z+k$. Then $p_{k}\left(z+z^{\prime}\right)=p_{0}\left(y+z^{\prime}\right)$ whenever either is defined. Since $z \notin \mathcal{O}(0)$, there exists $i_{0}$ such that $y_{i}=z_{i}$ for $i \geqq i_{0}$. Let

$$
U=\left\{\sum u_{i} n^{i} \mid u_{i}=0 \text { for } i<i_{0}\right\}
$$

Suppose that $u \in U$ and $z+u \in Z^{*}$. If $\omega=\pi^{-1}(z+u)$, then for each $i \geqq 0$, $\omega\left[-(z+u)^{(i)}, n^{i}\right]$ is either $\theta^{i}(0)$ or $\theta^{i}(1)$, and $p_{0}(z+u)=\omega_{0}$ is either $\theta^{i}(0)$ or $\theta^{i}(1)$ evaluated at $(z+u)^{(i)}$. Choose $i$ so that $(z+u)^{(i)} \in I_{\infty}$. Then $i \geqq i_{0}$ and $\theta^{i}(0)$ and $\theta^{i}(1)$ agree at place $(z+u)^{(i)}$. Therefore $p_{0}(z+u)$ is $\theta^{i}(0)$ evaluated at $(z+u)^{(i)}$. Similarly $(y+u)^{(i)} \in I_{\infty}$ and $p_{0}(y+u)$ is $\theta^{i}(0)$ evaluated at $(y+u)^{(i)}$.

By the observation made before the proof, $\theta^{i}(0)$ evaluated at $(z+u)^{(i)}$ is $\theta\left(s^{\prime}\right)$ evaluated at $(z+u)_{0}$ where $s^{\prime}$ is $\theta^{i-1}(0)$ evaluated at $(1 / n)\left[(z+u)^{(i)}-(z+u)_{0}\right]$. Applying the observation to $\theta^{i-1}(0)$, we see that $\theta^{i}(0)$ evaluated at $(z+u)^{(i)}$ is $\theta^{2}\left(s^{\prime \prime}\right)$ evaluated at $(z+u)_{0}+(z+u)_{1} n=(z+u)^{(1)}$ where $s^{\prime \prime}$ is $\theta^{i-2}(0)$ evaluated at

$$
\frac{1}{n}\left[\frac{1}{n}\left((z+u)^{(i)}-(z+u)_{0}\right)-(z+u)_{1}\right]=\frac{1}{n^{2}}\left[(z+u)^{(i)}-(z+u)^{(2)}\right] .
$$

Continuing in this manner we find that $\theta^{t}(0)$ evaluated at $(z+u)^{(i)}$ is $\theta^{t_{0}}(s)$ evaluated at $(z+u)^{\left(i_{0}\right)}$ where $s$ is $\theta^{i-i_{0}}(0)$ evaluated at $\left(1 / n^{i_{0}}\right)\left[(z+u)^{(i)}-(z+u)^{\left(i_{0}\right)}\right]$. Similarly $\theta^{i}(0)$ evaluated at $(y+u)^{(i)}$ is $\theta^{i_{0}}(\bar{s})$ evaluated at $(y+u)^{\left(i_{0}\right)}$ where $\bar{s}$ is $\theta^{i-i_{0}}(0)$ evaluated at $\left(1 / n^{i_{0}}\right)\left[(y+u)^{(i)}-(y+u)^{\left(i_{0}\right)}\right]$.

Note however that $(y+u)^{(i)}-(y+u)^{\left(i_{0}\right)}=(z+u)^{(i)}-(z+u)^{\left(i_{0}\right)}$ and therefore $s=\bar{s}$. Furthermore $(z+u)^{\left(i_{0}\right)}=z^{\left(i_{0}\right)}$ and $(y+u)^{\left(i_{0}\right)}=y^{\left(i_{0}\right)}$. Therefore for each $u \in U$ such 
that $z+u \in Z^{*}$, there exists $s=0$ or 1 such that

$$
\begin{array}{ll}
p_{0}(z+u)=\theta^{i}(s) & \text { evaluated at } z^{\left(i_{0}\right)}, \\
p_{k}(z+u)=\theta^{i_{0}}(s) & \text { evaluated at } y^{\left(i_{0}\right)} .
\end{array}
$$

Since $p_{0}$ is continuous at neither $y$ nor $z$, both $y$ and $z$ are in $J_{\infty}$. Hence $y^{\left(i_{0}\right)}$, $z^{\left(i_{0}\right)} \in J_{\infty}$ and $p_{0}(z+u)=p_{k}(z+u)$ or $p_{0}(z+u)=1-p_{k}(z+u)$ depending whether $\theta^{i_{0}}(0)$ evaluated at $z^{\left(i_{0}\right)}$ is the same or different from $\theta^{i}(0)$ evaluated at $y^{\left(i_{0}\right)}$. This is independent of $u$ and the lemma is proved.

If $z \notin Z^{*}$ and $z \in \mathcal{O}(0)$, then the method of the above proof can be used to show that locally $p_{k}$ depends on $p_{0}$ when $k \geqq 0$ and on $p_{-1}$ when $k<0$. In this case $z$ will have either two or four inverses. There will be four inverses if and only if $\theta(0)$ and $\theta(1)$ disagree at both ends.

It is now easy to show that $X^{*}$ is dense in $X$. Let $x \in X-X^{*}$. Choose a sequence $z_{n} \in Z^{*}$ such that $z_{n} \rightarrow \pi^{*}(x), p_{0}\left(z_{n}\right)=x\left(p_{0}\right)$ and, if necessary, $p_{-1}\left(z_{n}\right)=x\left(p_{-1}\right)$. Then $\pi^{*-1}\left(z_{n}\right) \rightarrow x$.

7. Topological classification of discrete substitutions. Let $\theta$ and $\theta^{\prime}$ be discrete substitutions having group systems $\left((Z(n), \tau), Z^{*}(\theta), p_{0}\right)$ and $\left(\left(Z\left(n^{\prime}\right), \tau\right), Z^{*}\left(\theta^{\prime}\right), p_{0}^{\prime}\right)$ respectively. The group systems are called isomorphic if there is an isomorphism $\psi:(Z(n), \tau) \rightarrow\left(Z\left(n^{\prime}\right), \tau\right)$ such that

(1) $\psi\left(Z^{*}(\theta)\right)=Z^{*}\left(\theta^{\prime}\right)$.

(2) For any convergent sequence $\left\{z_{k}\right\}$ in $Z^{*}(\theta),\left\{p_{0}\left(z_{k}\right)\right\}$ converges if and only if $\left\{p_{0}^{\prime}\left(\psi z_{k}\right)\right\}$ converges.

(3) For any convergent sequence $\left\{z_{k}\right\}$ in $Z^{*}(\theta),\left\{p_{-1}\left(z_{k}\right)\right\}$ converges if and only if $\left\{p_{-1}^{\prime}\left(\psi z_{k}\right)\right\}$ converges.

Note that condition (3) is superfluous unless either $\theta$ or $\theta^{\prime}$ disagrees at both ends.

TheOREM. Let $\theta$ and $\theta^{\prime}$ be discrete substitutions. Then $\left(\mathcal{O}_{\theta}, \sigma\right)$ and $\left(\mathcal{O}_{\theta^{\prime}}, \sigma\right)$ are isomorphic if and only if $\theta$ and $\theta^{\prime}$ have isomorphic group systems.

Proof. The "if" part follows from the construction of $\S 6$.

Suppose that $\varphi:\left(\mathcal{O}_{\theta}, \sigma\right) \rightarrow\left(\mathcal{O}_{\theta^{\prime}}, \sigma\right)$ is an isomorphism. Then $\varphi$ induces an isomorphism $\psi$ of the corresponding structure systems such that the following diagram commutes:

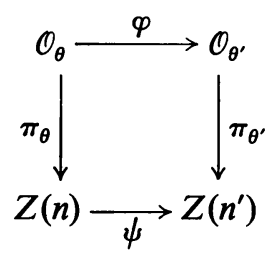

where $\pi_{\theta}$ and $\pi_{\theta^{\prime}}$ are the maps described in $\S 5$. A standard argument about cardinality of fibres shows that $\psi\left(Z^{*}(\theta)\right)=Z^{*}\left(\theta^{\prime}\right)$. Suppose that $\left\{z_{k}\right\}$ is a convergent sequence in $Z^{*}(\theta)$ such that $\left\{p_{0}\left(z_{k}\right)\right\}$ converges. Without loss of generality we may assume 
that $p_{0}\left(z_{k}\right)=0$ for all $k$. Let $\omega^{(k)}=\pi_{\theta}^{-1}\left(z_{k}\right)$; then $\omega_{0}^{(k)}=0$ for all $k$. Since the diagram commutes, $\varphi\left(\omega^{(k)}\right)=\pi_{\theta^{\prime}}^{-1}\left(\psi z_{k}\right)$ and in particular $\varphi\left(\omega^{(k)}\right)_{0}=p_{0}^{\prime}\left(\psi z_{k}\right)$. Since $\varphi$ is continuous, $\varphi\left(\omega^{(k)}\right)_{0}$ is eventually constant, that is, $p_{0}^{\prime}\left(\psi z_{k}\right)$ converges. The same argument using $\varphi^{-1}$ in place of $\varphi$ shows that if $\left\{p_{0}^{\prime}\left(\psi z_{k}\right)\right\}$ converges, then $\left\{p_{0}\left(z_{k}\right)\right\}$ also converges. The proof of condition (3) is exactly the same as the proof of condition (2).

Let $\theta$ be a discrete substitution of length $n$ with

$$
\theta(0)=a_{0} a_{1} \cdots a_{n-1}, \quad \theta(1)=b_{0} b_{1} \cdots b_{n-1} .
$$

If $a_{0}=b_{0}$, define a substitution $\zeta$ by

$$
\zeta(0)=a_{1} a_{2} \cdots a_{n-1} a_{0}, \quad \zeta(1)=b_{1} b_{2} \cdots b_{n-1} b_{0} .
$$

It is easy to see that $\mathcal{O}_{\theta}=\mathcal{O}_{\zeta}$, for $\zeta(0)$ appears in $\theta(0) \theta(1)$ and $\zeta(1)$ appears in $\theta(1) \theta(0)$. Therefore every block in $\mathcal{O}_{\zeta}$ appears in $\mathcal{O}_{\theta}$ and the sets are equal by minimality.

Define another substitution $\tilde{\theta}$ by $\tilde{\theta}(0)=\tilde{b}$ and $\tilde{\theta}(1)=\tilde{a}$. Then $\mathcal{O}_{\theta}$ and $\mathcal{O}_{\tilde{\theta}}$ are isomorphic via the mirror map $\omega \rightarrow \tilde{\omega}$.

A discrete substitution $\theta(\theta(0)=a, \theta(1)=b)$ is said to be in normal form if

(1) $a_{0} \neq b_{0}$.

(2) If $j=\min \left\{i \mid a_{i}=b_{i}\right\}$, then $a_{j}=b_{j}=0$.

The remarks just made show that two discrete substitutions of equal length having the same normal form have isomorphic substitution minimal sets. The converse yields the topological classification which we seek.

THEOREM. Let $\theta$ and $\theta^{\prime}$ be discrete substitutions of the same length. Then $\left(\mathcal{O}_{\theta}, \sigma\right)$ and $\left(\mathcal{O}_{\theta^{\prime}}, \sigma\right)$ are isomorphic if and only if $\theta$ and $\theta^{\prime}$ have the same normal form.

Proof. The proof is based on the isomorphism theorem for discrete substitutions proved earlier in this section. We shall outline the proof leaving the details to the reader.

Suppose that $\theta$ and $\theta^{\prime}$ are distinct substitutions of length $n$, both in normal form, and that $\left(\mathcal{O}_{\theta}, \sigma\right)$ and $\left(\mathcal{O}_{\theta^{\prime}}, \sigma\right)$ are isomorphic. Let $\psi:(Z(n), \tau) \rightarrow(Z(n), \tau)$ be the isomorphism which exhibits the isomorphism of their group systems. Note that any isomorphism $\varphi$ of $(Z(n), \tau)$ has the form $\varphi(z)=z+\bar{z}$ for some fixed $\bar{z} \in Z(n)$.

There are three ways in which $\theta$ and $\theta^{\prime}$ can fail to have the same normal form:

(i) $J_{1}(\theta) \neq J_{1}\left(\theta^{\prime}\right)$. In this case $\psi$ cannot map $Z^{*}(\theta)$ to $Z^{*}\left(\theta^{\prime}\right)$. This is proved by using the form of $\psi$ and the fact that $0 \in J_{1}(\theta) \cap J_{1}\left(\theta^{\prime}\right)$.

(ii) $I_{1}(\theta)=I_{1}\left(\theta^{\prime}\right)$ and $a_{i} \neq a_{i}^{\prime}$ for some $i \in I_{1}(\theta)$. In this case one of the convergence criteria (2) or (3) for isomorphism of group systems is not satisfied.

(iii) $I_{1}(\theta)=I_{1}\left(\theta^{\prime}\right)$ and $a_{i} \neq a_{i}^{\prime}$ for some $i \in J_{1}(\theta)$. If (ii) does not hold, then this case may be reduced to (ii) by considering the squares of the substitutions.

We suspect that there is a "normal form" theorem for two discrete substitutions whose lengths have the same prime factors, although we have been unable to establish one. 
8. Continuous substitutions. Suppose $\theta$ is a continuous substitution of length $n$, $\theta(0)=a, \theta(1)=\tilde{a}$. As in the case of a discrete substitution of length $n$, the structure system of $\left(\mathcal{O}_{\theta}, \sigma\right)$ is $(Z(n), \tau)$ (see [2]). We shall use the theory of discrete substitutions to find a homomorphism $\pi:\left(\mathcal{O}_{\theta}, \sigma\right) \rightarrow(Z(n), \tau)$ and to establish a classification of all continuous substitutions with a common length.

Define $\bar{\theta}$, the discrete substitution associated with $\theta$, by

$$
\bar{\theta}(0)=c_{0} c_{1} \cdots c_{n-1}, \quad \bar{\theta}(1)=d_{0} d_{1} \cdots d_{n-1},
$$

where $c_{i}=d_{i}=a_{i}+a_{i+1}(\bmod 2)(i=0,1, \ldots, n-2), c_{n-1}=\tilde{d}_{n-1}=a_{n-1}+a_{1}(\bmod 2)$. Note that in general, $\bar{\theta}$ does not come out in normal form.

Define $f_{\infty}:(\Omega, \sigma) \rightarrow(\Omega, \sigma)$ by $f_{\infty}(\omega)_{i}=\omega_{i}+\omega_{i+1}(\bmod 2)$. (In the terminology of [4], $f_{\infty}$ is the endomorphism of $(\Omega, \sigma)$ obtained from the block map $f\left(x_{1}, x_{2}\right)$ $=x_{1}+x_{2}$.) It is easy to see that the restriction of $f_{\infty}$ to $\mathcal{O}_{\theta}$ is a homomorphism of $\left(\mathcal{O}_{\theta}, \sigma\right)$ onto $\left(\mathcal{O}_{\bar{\theta}}, \sigma\right)$ which is exactly two-to-one and is a local homeomorphism. The map $\pi$ is given by $\bar{\pi} \circ f_{\infty}$ where $\bar{\pi}$ is the map constructed in $\S 5$ for the discrete substitution $\bar{\theta}$. We remark that the chain $\left(\mathcal{O}_{\theta}, \sigma\right) \rightarrow\left(\mathcal{O}_{\bar{\theta}}, \sigma\right) \rightarrow(Z(n), \tau)$ is an example of the decomposition theory for point-distal flows due to Veech [10].

If $a_{0}=0$, then both $\theta(0)$ and $\theta^{2}(0)$ begin with 0 , and if $a_{0}=1$, then $\theta^{2}(0)$ begins with 0 . With this observation in mind, we say that a continuous substitution is in normal form if $a_{0}=0$.

Theorem. Let $\theta$ and $\theta^{\prime}$ be continuous substitutions of the same length. Then $\left(\mathcal{O}_{\theta}, \sigma\right)$ and $\left(\mathcal{O}_{\theta^{\prime}}, \sigma\right)$ are isomorphic if and only if $\theta$ and $\theta^{\prime}$ have the same normal form.

The proof is routine using the normal form theorem for discrete substitutions and is left to the reader.

9. Examples. In doing computations involving substitutions it is useful to think of the group $Z(n)$ as identified with a unit interval having two points at each $n$-adic rational via the map $\sum z_{i} n^{i} \rightarrow \sum z_{i} n^{-i-1}$.

1. Let $a=01, b=00$. The group is $Z(2)$ which we identify with the unit interval (with two points at each dyadic rational) as above. Then $J_{\infty}$ is the singleton $(.111 \ldots)$ and the function $p_{0}$ is zero on the intervals $\left[1-1 / 2^{2 k+1}, 1-1 / 2^{2 k}\right]$ and one on the intervals $\left[1-1 / 2^{2 k}, 1-1 / 2^{2 k-1}\right]$. The ambiguity occurs since each point in the orbit of $(.111 \ldots)$ must be split into two points in $\mathcal{O}_{\theta}$.

2. Let $a=011, b=101$. The group is $Z(3)$ and $J_{\infty}=\left\{\sum z_{i} n^{i} \mid z_{i}=0\right.$ or 1$\}$. Then $E$ consists of all points $\sum z_{i} n^{i}$ such that $z_{i}=0$ or 1 for all but finitely many $i$ together with the orbit of 0 . That is $E=\left\{\sum z_{i} n^{i} \mid z_{i}=0\right.$ or 1 for all but finitely many $i$ or $z_{i}=2$ for all but finitely many $i\}$. Each point in $E$ has two inverses. Note that $-1 \in E$ since $-1=\sum 2 \cdot 3^{i}$.

3. Let $a=001, b=100$. Here $\pi^{-1}(z)$ consists of four points for each $z \in \mathcal{O}(0)$ thus the functions $p_{0}$ and $p_{-1}$ are "independent". If $z \in E-\mathcal{O}(0)$ then $\pi^{-1}(z)$ consists of two points. Note that $E-\mathcal{O}(0) \neq \varnothing$ for it contains the point $\sum z_{i} 3^{i}$ where $z_{i}=0$ for $i$ odd and $z_{i}=2$ for $i$ even. 


\section{REFERENCES}

1. Robert Ellis and W. H. Gottschalk, Homomorphisms of transformation groups, Trans. Amer. Math. Soc. 94 (1960), 258-271. MR 23 \#A960.

2. W. H. Gottschalk, Substitution minimal sets, Trans. Amer. Math. Soc. 109 (1963), 467-491. MR 32 \#8325.

3. W. H. Gottschalk and G. A. Hedlund, Topological dynamics, Amer. Math. Soc. Colloq. Publ., vol. 36, Amer. Math. Soc., Providence, R. I., 1955. MR 17, 650.

4. G. A. Hedlund, Endomorphisms and automorphisms of the shift dynamical system, Math. Systems Theory 3 (1969), 320-375. MR 41 \#4510.

5. K. Jacobs and M. Keane, 0-1-sequences of Toeplitz type, $Z$. Wahrscheinlichkeitstheorie und Verw. Gebiete 13 (1969), 123-131. MR 41 \#426.

6. M. Keane, Generalized Morse sequences, Z. Wahrscheinlichkeitstheorie und Verw. Gebiete 10 (1968), 335-353. MR 39 \#406.

7. Benjamin G. Klein, Homomorphisms of symbolic dynamical systems, Dissertation, Yale University, New Haven, Conn., 1968.

8. J. Neveu, Sur les suites de Toeplitz, Z. Wahrscheinlichkeitstheorie und Verw. Gebiete 13 (1969), 132-134.

9. J. C. Oxtoby, Ergodic sets, Bull. Amer. Math. Soc. 58 (1952), 116-136. MR 13, 850.

10. W. A. Veech, Point-distal flows, Amer. J. Math. 92 (1970), 205-242.

Wesleyan University, Middletown, Connecticut 06457

Yale University, New Haven, Connecticut 06520 